\title{
Aplicación y evaluación de la gamificación en la docencia universitaria: una experiencia docente
}

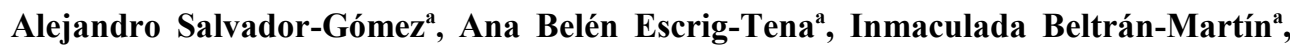 \\ Georgiana-Alexandra Badoiu ${ }^{a}$ \\ Universitat Jaume I, Castellón de la Plana, España, alsalvad@uji.es' bescrigt@uji.es, ${ }^{c}$ ibeltran@uji.es, \\ dalexandra.badoiu@uji.es
}

\begin{abstract}
Resumen
En este trabajo se presenta la experiencia de diseñar e implementar una estrategia de gamificación en la docencia universitaria. El objetivo final de esta innovación era combinar mecánicas y dinámicas del juego, de tal forma que, en conjunto, formaran una herramienta docente capaz de repercutir en la motivación del alumnado hacia la participación y mejora en su desempeño. La información obtenida de los estudiantes, a través de cuestionarios y entrevistas semiestructuradas, muestra, por una parte, que la estrategia de gamificación fue percibida como útil para su aprendizaje y atractiva para su experiencia educativa, y por otra, que mejora la participación y el rendimiento del estudiante en la asignatura.
\end{abstract}

Palabras clave: gamificación, docencia, utilidad percibida, motivación

\section{Introducción}

El método de enseñanza tradicional de clase magistral está en entredicho. Por una parte, la transmisión de conocimiento unidireccional entra en conflicto con el perfil actual del alumnado, caracterizado por demandar información de forma ágil e inmediata, y por tener preferencia clara hacia instrucciones lúdicas y participativas (Prensky, 2010). Por otra, la emergencia sanitaria COVID-19 ha mostrado la necesidad de adaptar el proceso de enseñanza aprendizaje a entornos virtuales (Cóndor-Herrera, 2020).

La gamificación, entendida como el uso de elementos del juego en contextos no lúdicos (Deterding et al., 2011) es una herramienta con capacidad para lograr esta transformación educativa. En primer lugar, porque la aplicación de la gamificación está estrechamente relacionada con el uso de la informática y de la formación a distancia (Raymer, 2011). Y en segundo lugar, porque tiene el potencial de conectar con el nuevo perfil del estudiante, ya que a la vez que entretiene y desafía, promueve el aprendizaje mediante la acción, implicación y atención del alumnado (Kapp, 2012). No obstante, la mera inclusión de 
elementos del juego (ej. puntos, insignias o clasificaciones), no garantiza el éxito de la gamificación (Hamari, 2013; Seaborn \& Fels, 2015). Si no se presta especial atención al proceso de diseño e implementación de la estrategia (Oliva, 2017) se incurre en la posibilidad de obtener resultados contraproducentes, como por ejemplo, menor participación en clase (Domínguez et al., 2013) o menor satisfacción y motivación del alumnado a lo largo del curso (Hanus \& Fox, 2015). Por tanto, es primordial, en primer lugar, definir los pilares en los que se fundamenta la estrategia gamificada, por ejemplo el tipo de audiencia, los objetivos o los recursos disponibles, para posteriormente, entender qué elementos del juego son apropiados incluir en el sistema (Werbach y Hunter, 2012).

A continuación presentamos la experiencia docente de diseñar e implementar una estrategia gamificada durante el curso 2019-2020 en la asignatura de Gestión de Calidad y RRHH impartida en el $4^{\circ}$ curso del grado de RRLL y RRHH en la Universitat Jaume I.

\section{Exposición de la metodología de diseño y aplicación}

El proyecto de gamificación comienza con el proceso (sintetizado en la tabla 1) de preparar y diseñar la estrategia. Siguiendo a Werbach y Hunter (2012), para implementar exitosamente la gamificación, es necesario, en primer lugar, a) definir los objetivos y actitudes que se pretenden fomentar en la audiencia; b) analizar el contexto y las características de la audiencia, y c) establecer los recursos y actividades que se van a utilizar para el desarrollo de la gamificación.

Tabla 1. Fase de preparación y diseño del sistema de gamificación

\begin{tabular}{|l|l|}
\hline Paso 1: Análisis inicial \\
\hline Objetivos & $\begin{array}{l}\text { Desarrollo de una herramienta docente útil para el aprendizaje del alumnado y que mejore su } \\
\text { experiencia educativa }\end{array}$ \\
\hline $\begin{array}{l}\text { Actitudes } \\
\text { deseadas }\end{array}$ & $\begin{array}{l}\text { Motivación y compromiso por participar en clase, por mejorar su rendimiento y por trabajar } \\
\text { en equipo }\end{array}$ \\
\hline $\begin{array}{l}\text { Perfil } \\
\text { alumnado }\end{array}$ & $\begin{array}{l}\text { Jóvenes de entre } 21 \text { y } 25 \text { años familiarizados con el uso de tecnologías (móviles, ordenadores, } \\
\text { tabletas....) y videojuegos. }\end{array}$ \\
\hline $\begin{array}{l}\text { Análisis } \\
\text { contextual }\end{array}$ & $\begin{array}{l}\text { 35 alumnos. Último año del grado. Realizan simultáneamente prácticas externas en empresas } \\
\text { y trabajo final de grado }\end{array}$ \\
\hline $\begin{array}{l}\text { Actividades en } \\
\text { la asignatura }\end{array}$ & $\begin{array}{l}\text { Metodología de Aula Inversa. Actividades individuales online (previas a clase y adicionales). } \\
\text { Actividades grupales (en clase) }\end{array}$ \\
\hline $\begin{array}{l}\text { Recursos } \\
\text { disponibles }\end{array}$ & $\begin{array}{l}\text { Aula virtual (Moodle) con capacidad de inserción de insignias. Google Drive. Dispositivos } \\
\text { móviles, tabletas y ordenadores. }\end{array}$ \\
\hline Paso 2: Selección e integración de elementos del juego \\
\hline Mecánicas & Puntos, rangos, retos, hall of fame, trabajo en equipo, insignias, progreso \\
\hline Dinámicas & Recompensas, estatus, logro, autonomía, control, competición, socialización \\
\hline
\end{tabular}


En segundo lugar, se deben seleccionar e incluir en el sistema los elementos del juego apropiados, es decir, las mecánicas y dinámicas. Mientras que las mecánicas son los elementos del juego que rigen las reglas del mismo y permiten traducir las acciones del jugador en resultados visibles (como por ejemplo, los puntos o insignias) las dinámicas son aquellos efectos, motivaciones y deseos que se pretenden generar en el participante (por ejemplo, el logro o la socialización) (Bunchball, 2010). A continuación, detallamos el diseño del sistema y la interrelación entre los elementos del juego, actividades y recursos.

Puntos. En nuestro caso consideramos el sistema de evaluación como elemento fundamental para gamificar la asignatura. Este elemento es, sin duda, la base del proceso formativo a través del cual los alumnos prestan mayor atención. De forma resumida, establecimos un sistema de 1150 puntos. Los primeros 1000 puntos se corresponden con el propio sistema de evaluación. Por ejemplo, las actividades de evaluación continua (que a su vez consistían en actividades individuales previas a la clase y actividades grupales a desarrollar en la sesión de clase) con un peso del $40 \%$, el trabajo grupal final con un peso del $20 \%$ y el examen con un peso del $40 \%$ se traducía dentro del sistema gamificado, en 400, 200 y 400 puntos respectivamente. Por otra parte, con el fin de fomentar en el alumnado una participación constante, rendimiento grupales excelentes y el interés de ampliar el conocimiento de la asignatura más allá de los establecidos por la guía docente, destinamos 150 puntos en formato de logros y retos vinculados a la obtención de insignias.

Rangos. A partir del sistema de puntos, se diseña un sistema compuesto por 20 rangos que busca ser un elemento gráfico motivante a través del cual el alumnado pueda observar su progresión en el curso. Con el fin de "enganchar" al alumnado desde su comienzo, se establece un sistema incremental, a través del cual los primeros rangos eran sencillos de obtener, pero a medida que el alumnado avanzaba, la cantidad de puntos necesarios para alcanzar rangos superiores incrementan. También, con el fin de promover un sentimiento de prestigio o estatus, se otorgó a cada rango una "palabra" que describe su progreso en la asignatura. En cuanto a su diseño (Figura 1), seguimos estrategias similares a los juegos de ordenador más populares, como puede ser el League of Legends o World of Warcraft. En todos ellos, el componente visual tiene coherencia con el rango alcanzado. Es decir, el aspecto visual es más elaborado en rangos superiores.

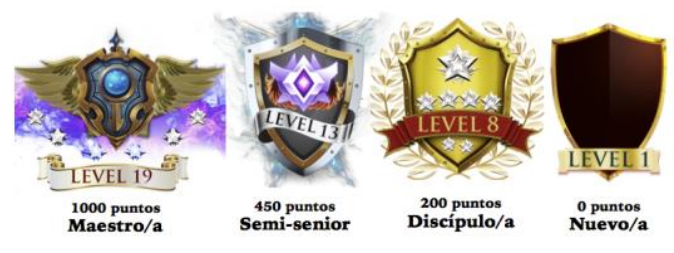

Fig. 1. Ejemplos de rangos utilizados en el sistema

Insignias y retos. Como complemento al sistema de rangos, e integrados en el sistema de puntos, se diseñaron tres tipos de insignias, asociadas a retos preestablecidos. 


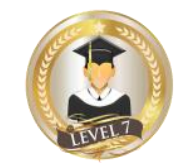

Insignias de participación

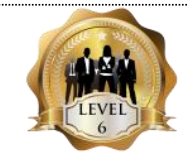

Insignias de equipo

Cada alumno que asistía a clase y participaba en la misma (a través de las actividades de clase, correcciones, etc) recibía un punto por sesión. Dependiendo del número de participaciones, el alumno conseguiría alcanzar una insignia u otra. La insignia de mayor nivel (7) se obtenía si el alumno había conseguido el reto de asistir a todas las clases y había participado en ellas.

Las actividades en la sesiones presenciales eran de carácter grupal. A través de estas insignias se trataba de fomentar un rendimiento excelente como equipo. En cada sesión, se otorgaban puntos a los miembros del equipo según los retos alcanzados. Por ejemplo, si la tarea entregada era excelente, y habían asistido todos los miembros se otorgaban 2 puntos, $\mathrm{La}$ insignia de mayor nivel (6) se otorgaba si se lograba alcanzar 80 puntos.

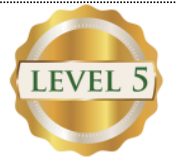

Estas insignias buscaban fomentar el rendimiento del alumnado en las actividades previas y su compromiso con la asignatura. Para poder optar a estas insignias, el alumno debía superar las tareas previas a la clase. Al superar una tarea, se desbloqueaba la opción de realizar una tarea adicional Insignias de vinculada con la gestión de la calidad. Si el alumno superaba todas las compromiso actividades previas (10 en total), y realizaba todas las tareas adicionales, obtendría la insignia de nivel 5 , y por tanto, la de mayor nivel.

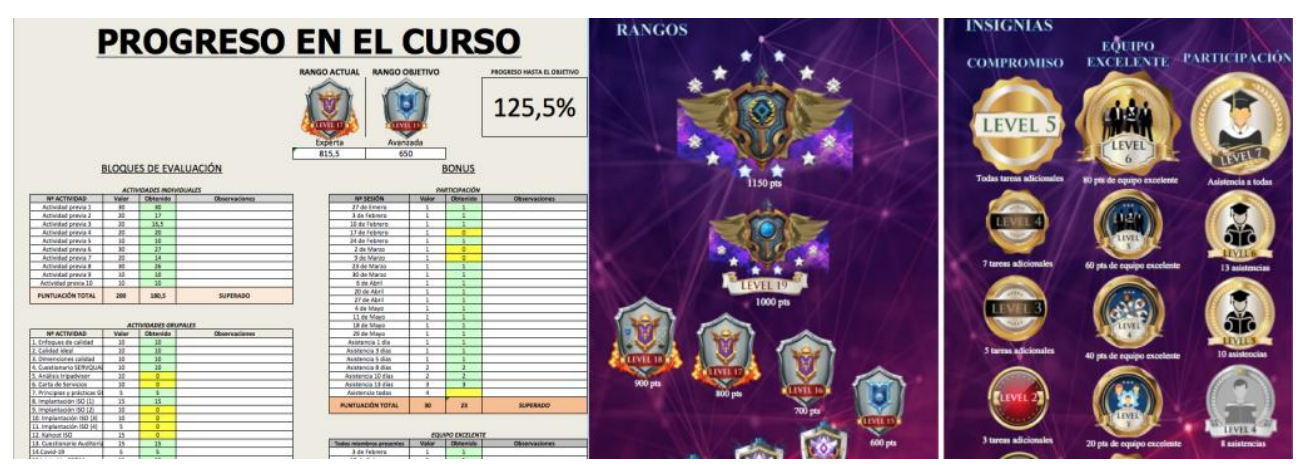

Fig. 2. Perfil Excel compartido entre el equipo docente y el alumno

Perfil Excel. Como elemento de engranaje, se diseña un "perfil individual Excel" a través del cual el alumnado visualizaba cada puntuación obtenida en cada una de las tareas. El perfil Excel, representado en la figura 2, se comparte entre el equipo docente y el alumno a través de Google Drive. Con este elemento se pretendía fomentar un ambiente de autosuperación dotando al alumnado de un mayor control y autonomía sobre su rendimiento. Para fomentar estos aspectos, incluimos un porcentaje de progreso a través del cual el alumnado visualizaba su progreso hacia el objetivo marcado al inicio de curso. 
Hall of fame. El equipo docente propone a los 5 alumnos con mayor rango participar en el Hall of Fame anual 2019/2020 presentado en el curso siguiente. Este elemento pretende, por una parte, reconocer a los alumnos de mayor rendimiento, fomentando la sensación de logro y perpetuidad y, por otra, ser un elemento de motivación para los alumnos venideros.

A continuación, y a modo de resumen, la figura 3 muestra las distintas etapas y acciones que el equipo docente ha realizado para implementar el sistema de gamificación

\section{FASE DE PUESTA EN MARCHA}

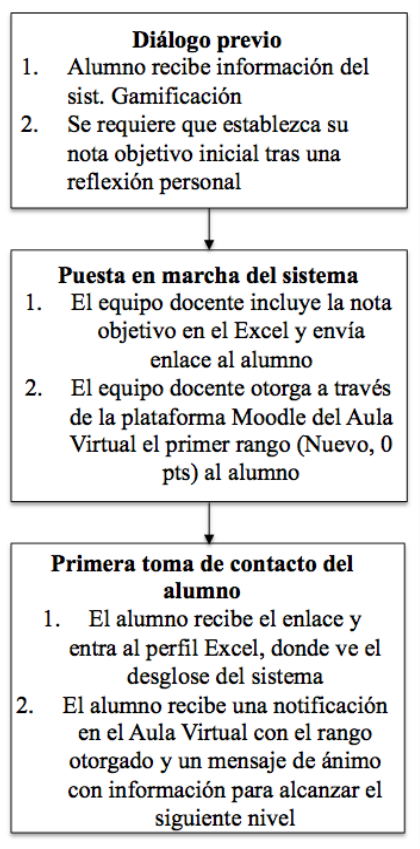

\section{FASE DE MANTENIMIENTO}

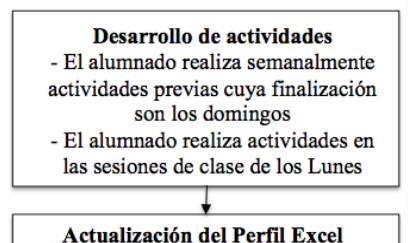

El equipo docente, cada Lunes, actualiza el perfil Excel de cada alumno con la notas obtenidas en cada actividad y una observación

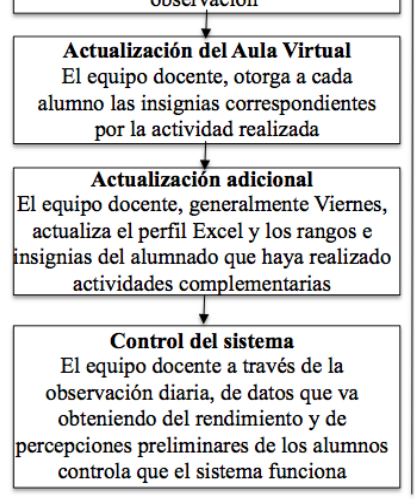

\section{FASE DE CONCLUSIÓN}

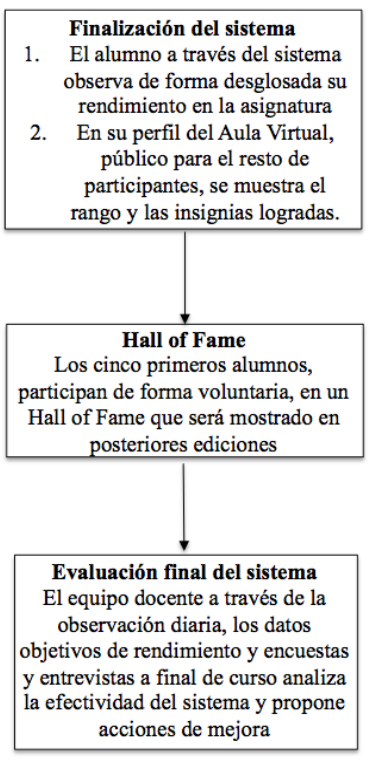

Fig. 3. Fases en la aplicación del sistema de gamificación

\section{Resultados obtenidos de evaluar el sistema de gamificación}

Con el fin de conocer de forma descriptiva la percepción del alumnado sobre el sistema de gamificación, se realizó una encuesta a final de curso. El cuestionario incluía, por una parte, cuestiones para que el alumnado valorara si el sistema de gamificación, y sus elementos, habían sido útiles para su aprendizaje, y por otra, si mejoraban su experiencia como estudiante en comparación con otras asignaturas. Todos los ítems utilizados utilizaban una escala Likert de 7 puntos. De 35 alumnos (31 activos), se obtuvo una muestra de 28 alumnos. Con el fin de contrastar la información obtenida de la propia actividad del alumnado (asistencia, participación y rendimiento) y de los cuestionarios, realizamos 2 entrevistas semiestructuradas grupales y 5 individuales. En total obtenemos información cualitativa de 11 alumnos. Todas las entrevistas fueron grabadas en vídeo y transcritas. 
De forma general, los datos obtenidos de participación, asistencia y rendimiento de los alumnos son muy buenos. La asistencia y participación media en actividades presenciales fue del $75 \%$, la participación media en actividades previas (fuera del aula) fue del $87 \%$ y el rendimiento medio del alumnado fue 1 punto superior respecto a los objetivos personales inicialmente planteados por cada uno de los alumnos (ver figura 3). Para comprobar que efectivamente, el sistema de gamificación ha podido incidir en estos resultados analizamos la información de las encuestas y de las entrevistas.

Los resultados de las encuestas muestran la efectividad del diseño y la implementación del sistema. En el primer bloque de la Tabla 1, se observa que el alumnado percibe que el sistema ha sido útil para su aprendizaje (media de 6,5). Entre sus elementos, destacamos la valoración que obtiene el perfil Excel $(6,7)$, el porcentaje de progreso $(6,6)$ y la aplicación de recompensas $(6,6)$. Los equipos de trabajo, en cambio, fue el elemento con menor valoración $(6,3)$ y mayor disparidad de puntuación. En el segundo bloque, observamos que los estudiantes valoran que el sistema de gamificación, en comparación con otros cursos, ha sido atractivo para su experiencia educativa $(6,4)$. En este caso, el perfil Excel $(6,6)$ y las recompensas $(6,6)$ han obtenido las puntuaciones mas altas. En este bloque, los equipos de trabajo también obtuvieron una menor valoración (6), posiblemente su utilización es habitual en otras asignaturas del grado.

Tabla 1. Estadísticos descriptivos de la utilidad percibida y mejora de la experiencia educativa $(\mathbf{N}=\mathbf{2 8})$

\begin{tabular}{|l|c|c|c|c|c|c|c|c|}
\cline { 2 - 9 } \multicolumn{1}{c|}{} & \multicolumn{4}{c|}{ Utilidad en el aprendizaje } & \multicolumn{3}{c|}{ Mejora en la experiencia educativa } \\
\hline \multicolumn{1}{c|}{ Elementos del sistema } & Media & Mediana & d.t & $\mathbf{\%}$-7 & Media & Mediana & d.t & \% 6-7 \\
\hline 1. Porcentaje de progreso & 6,6 & 7 & 0.62 & $92 \%$ & 6,4 & 7 & 0.69 & $90 \%$ \\
\hline 2. Perfil Excel & 6,7 & 7 & 0.59 & $92 \%$ & 6,6 & 7 & 0.62 & $92 \%$ \\
\hline 3. Equipos de trabajo & 6,3 & 7 & 1.27 & $83 \%$ & 6 & 6 & 1.29 & $68 \%$ \\
\hline 4. Rangos e insignias & 6,4 & 7 & 0.86 & $83 \%$ & 6,4 & 7 & 0.95 & $86 \%$ \\
\hline 5. Sistema de puntos & 6,4 & 7 & 0.83 & $86 \%$ & 6,5 & 7 & 0.79 & $90 \%$ \\
\hline 6. Recompensas & 6,6 & 7 & 0.68 & $90 \%$ & 6,6 & 7 & 0.62 & $92 \%$ \\
\hline Media sistema & $\mathbf{6 , 5}$ & $\mathbf{7}$ & $\mathbf{0 . 8 4}$ & $\mathbf{8 7 \%}$ & $\mathbf{6 , 4}$ & $\mathbf{7}$ & $\mathbf{0 . 8 7}$ & $\mathbf{8 6 \%}$ \\
\hline
\end{tabular}

Por otro lado, la información obtenida a través de las entrevistas revela que el sistema ha tenido un efecto positivo en la motivación del alumnado por participar en la asignatura y por realizar mayores esfuerzos en las actividades. Observamos que, dependiendo del alumno, la motivación se activa por dinámicas diferentes o por una combinación de las mismas (ej. feedback, logro...). Algunos comentarios del estudiantado así lo confirman:

Estudiante 1. "La verdad es que es super motivante, cuando hacia las actividades individuales y adicionales, a toda hora estaba mirando el Excel para ver si habias puesto la nota para motivarme. [...] es una forma de, no sé, lo quieres ver completo, lo quieres ver con puntuación, quieres superarte, y es una forma de realmente motivarte al máximo y creo que eso es lo mejor de la asignatura." 
Estudiante 11. "A mí el tema de la gamificación me ha encantado. Yo me picaba con mis compañeros, porque nos preguntabamos ¿Cuánto tienes? Yo 400...ah si, pues yo voy a hacer esto, voy a hacer lo otro y al final nos poníamos"... "Te animaba a trabajar mejor y a hacer más."

Estudiante 7. "A mi me encanta, la verdad. Me motiva bastante conseguir distinciones. El haber ido a todas las clases tienes insignias que a lo mejor no tendrías si no vas, el haber hecho todas las faenas extras te da una insignia. Porque llegué a pensar, esta actividad no sé si hacerla porque por 0.04 tampoco pasa nada, pero claro, hay una insignia que es haz todas las actividades, entonces si no las hago no las voy a tener así, que la voy a hacer. Es positivo."

También observamos que el alumnado percibe que el sistema es compacto y coherente. Entre sus elementos, destacamos que el perfil Excel y la barra de progreso han sido de gran utilidad para su aprendizaje y, por otra parte, que el diseño y sistema de rangos e insignias era muy atractivo y mejoraba la experiencia educativa.

Estudiante 5. "Yo me quedo con todo en general porque una cosa deriva a la otra porque si estás haciendo tu trabajo con tus compañeros pues optas a un bonus y a su vez vas subiendo de nivel, y consigues la insignia. Entonces digamos que todo está muy relacionado. Si tuviera que quedarme con una sola cosa, en todo caso sería el porcentaje hacia tu objetivo, que te hace verlo mucho mas claro si vas por el buen camino o no."

Estudiante 4. "Yo me quedaría con el porcentaje de progreso hasta el objetivo teniendo lo de rango actual y rango objetivo porque asi también realmente eres consciente de donde estás, lo que te queda o lo que sabes, y tu también te puedes autogestionar."

Estudiante 7. "Ver los porcentajes de esa forma, el tener todo desglosado, y decir mira, hasta aqui he llegado y tener el feedback tan constante. Porque claro, muchas veces (haciendo alusión a otras experiencias) haces actividades en clase, haces prácticas y a lo mejor el profesor va evaluando pero tu para empezar no ves qué resultados estás teniendo, y aqui si que los ves y además están desglosados por diferentes categorías, ves el límite, ves los puntos. Entonces, tienes claro siempre lo que tienes que hacer o por donde vas."

\section{Conclusiones}

Los resultados obtenidos nos permiten concluir la efectividad del diseño y aplicación del sistema gamificado. A través del mismo, el alumnado percibió que participaba de forma activa en su aprendizaje y que, gracias a la retroalimentación constante que recibió por parte del sistema (rangos, insignias, perfil..), tenía gran control y autonomía sobre su rendimiento. Como consecuencia, el alumnado consideró esta herramienta útil para su aprendizaje y una experiencia educativa muy positiva. La información cualitativa refleja que el sistema motiva al alumnado, animándoles a participar y a esforzarse más en las actividades. Desde el punto de vista docente, existen ciertas ventajas e inconvenientes que cabría resaltar. En cuanto a las ventajas, destacamos el alto nivel de gratificación y motivación en el trabajo como docente. Por una parte, se disfruta aplicando un sistema de 
gamificación. Por otra, el compromiso de retroalimentación que se adquiere es alto y constante, lo cual genera valoraciones positivas por parte del alumnado respecto a la implicación docente. También consideramos que esta herramienta ha sido un complemento perfecto en la aplicación del aula inversa, y de ayuda en la inesperada situación de emergencia sanitaria COVID-19, ya que ha fomentado la participación del estudiante fuera del aula. En cuanto a sus inconvenientes destacamos especialmente dos. El primero, es que el diseño y mantenimiento del sistema implica una carga de trabajo abundante ya que, para ser efectivo, requiere de una retroalimentación individual constante. En relación con el segundo inconveniente, consideramos que en asignaturas con gran cantidad de estudiantes, la aplicación del sistema puede ser inviable a menos que algunos procesos se automaticen.

\section{Referencias}

Bunchball. Inc. (2010). Gamification 101: An introduction to the use of game dynamics to influence behavior. White paper. Author.

Cóndor-Herrera, O. (2020). Educar en tiempo de COVID-19. CienciAmérica, 9(2). pp. 31-37.

Deterding, S., Dixon, D., Khaled, R. \& Nacke, L. (2011). From game design elements to gamefulness: defining gamification. In Proceedings of MindTrek.

Domínguez, A., Saenz-de-Navarrete, J., De-Marcos, L., Fernández-Sanz, L., Pagés, C. \& MartínezHerráiz, J.J. (2013). Gamifying learning experiences: practical implications and outcomes. Computers \& Education, 63, pp. 380-392.

Hamari, J. (2013). Transforming homo economicus into homo ludens: a field experiment on gamification in a utilitarian peer-to-peer trading service. Electronic Commerce Research and Applications, 12, pp. 236-245.

Hanus, M. D. \& Fox, J. (2015). Assessing the effects of gamification in the classroom: A longitudinal study on intrinsic motivation, social comparison, satisfaction, effort, and academic performance. Computers \& Education, 80, pp. 152-161.

Kapp, K. M. (2012). The gamification of learning and instruction. San Francisco: Wiley.

Oliva, H. (2017). La gamificación como estrategia metodológica en el contexto educativo universitario. Realidad y Reflexión, 44, pp. 29-47.

Prensky, M. (2010), "Nativos e Inmigrantes Digitales", Cuadernos SEK 2.0, Institución Educativa SEK, Distribuidora SEK, S.A. pp. 1-21.

Raymer, R. (2011). Gamification: using game mechanics to enhance e learning. eLearn, (9), 3.

Seaborn, K. \& Fels, D. I. (2015). Gamification in theory and action: A survey. International Journal of Human-Computer Studies, 74, pp.14-31.

Werbach, K., \& Hunter, D. (2012). For the Win: How Game Thinking Can Revolutionize Your Business. Philadelphia: Wharton Digital Press. 